University of Nebraska - Lincoln

DigitalCommons@University of Nebraska - Lincoln

Faculty Publications from the Department of Electrical \& Computer Engineering, Department Electrical and Computer Engineering

2012

\title{
Optical properties of cobalt slanted columnar thin films passivated by atomic layer deposition
}

\author{
Daniel Schmidt \\ University of Nebraska-Lincoln, schmidt@huskers.unl.edu \\ Eva Schubert \\ University of Nebraska-Lincoln, efranke3@unl.edu \\ Mathias Schubert \\ University of Nebraska-Lincoln, mschubert4@unl.edu
}

Follow this and additional works at: https://digitalcommons.unl.edu/electricalengineeringfacpub

Part of the Electrical and Computer Engineering Commons

Schmidt, Daniel; Schubert, Eva; and Schubert, Mathias, "Optical properties of cobalt slanted columnar thin films passivated by atomic layer deposition" (2012). Faculty Publications from the Department of Electrical and Computer Engineering. 192.

https://digitalcommons.unl.edu/electricalengineeringfacpub/192

This Article is brought to you for free and open access by the Electrical \& Computer Engineering, Department of at DigitalCommons@University of Nebraska - Lincoln. It has been accepted for inclusion in Faculty Publications from the Department of Electrical and Computer Engineering by an authorized administrator of DigitalCommons@University of Nebraska - Lincoln. 


\title{
Optical properties of cobalt slanted columnar thin films passivated by atomic layer deposition
}

\author{
Daniel Schmidt, ${ }^{\text {a) }}$ Eva Schubert, and Mathias Schubert \\ Department of Electrical Engineering and Nebraska Center for Materials and Nanoscience, \\ University of Nebraska-Lincoln, Lincoln, Nebraska 68588-0511, USA
}

(Received 14 November 2011; accepted 12 December 2011; published online 6 January 2012)

\begin{abstract}
Optical properties of passivated metal slanted columnar thin films from cobalt within the visible spectral region are reported. Glancing angle deposition is utilized to grow slanted nanocolumns which have been conformally coated with $\mathrm{Al}_{2} \mathrm{O}_{3}$ by a subsequent atomic layer deposition process. A generalized anisotropic Bruggeman effective medium approximation has been employed to analyze spectroscopic generalized ellipsometry data. The modified homogenization approach allows for determination of biaxial (monoclinic) optical and structural properties as well as fractions of three film constituents. The conformal alumina passivation layer preserves the pristine metal character of the nanostructures and prevents oxidation and aging effects. (C) 2012 American Institute of Physics. [doi:10.1063/1.3675549]
\end{abstract}

Three-dimensionally shaped metal sculptured thin films made by glancing angle deposition (GLAD) resemble an artificial material class with interesting physical properties. ${ }^{1}$ However, in order to exploit optical and magneto-optical (magnetic) properties for possible applications, the asdeposited intrinsic material characteristics such as conductivity or ferromagnetic properties need to be preserved. ${ }^{2,3}$ Hence, a thin homogeneous passivation shell is desired to especially prevent alterations due to material oxidation. It has been shown that atomic layer deposition (ALD) is an excellent technique to conformally coat complex nanostructures and sculptured thin films. ${ }^{4,5}$

In general, the characterization of material properties and quantification of material fractions of complex anisotropic thin films is a challenge. Non-destructive optical investigations, in particular, generalized ellipsometry is an excellent technique for probing anisotropic physical properties. However, the indirect method requires adequate mathematical model descriptive systems to match experimentally acquired data sets. Previously, an anisotropic Bruggeman effective medium approximation (AB-EMA) was employed to determine optical properties of metal slanted columnar thin films (SCTFs) in the visible spectral range. However, models were limited to uniaxial ${ }^{6}$ or orthorhombic descriptions with a maximum of two different constituents. ${ }^{7-9}$ Recently, we have shown that such an AB-EMA approach also leads to a very good approximation of structural and physical properties of anisotropic Co SCTFs in the THz spectral range. ${ }^{10}$

In this letter, we focus on the determination and quantification of a thin conformal $\mathrm{Al}_{2} \mathrm{O}_{3}$ layer on top of $\mathrm{Co}$ nanocolumns. It is evaluated how the alumina layer serves as a passivation layer thereby preserving the as-deposited optical properties of the Co SCTF. An augmented AB-EMA optical model approach is introduced to accurately describe the anisotropic optical response within the visible spectral range of a thin film comprising three material constituents (Co, $\mathrm{Al}_{2} \mathrm{O}_{3}$, and air). This approach leads to the determination of

a)Electronic mail: schmidt@huskers.unl.edu. structural parameters in excellent agreement to complementary techniques and reference samples.

Slanted nanocolumns from cobalt were deposited by electron-beam GLAD in a custom-built ultra-high vacuum chamber onto a low-doped n-type (001) silicon substrate at a deposition angle of $85^{\circ}$. Details about the growth process can be found elsewhere. ${ }^{11}$ Immediately after the growth process, the sample was transferred to the ALD reactor (Fiji 200, CambridgeNanoTech Inc.). The subsequent thermal $\mathrm{Al}_{2} \mathrm{O}_{3}$ process $^{12}$ of 60 cycles was carried out at a substrate temperature of $60^{\circ} \mathrm{C}$ in order to avoid sample property alterations by annealing effects. Both precursors, trimethylaluminum and nanopure water, were pulsed at $60 \mathrm{~ms}$ each followed by a $30 \mathrm{~s}$ purge time, which warrants a homogeneous and conformal coating of the columns with an aspect ratio of approximately 9. Note that even though ALD is a wellknown technique to achieve highly conformal coatings of high aspect ratios, the uniformity is affected by non-ideal sample areas where columns are partially in contact. A schematic drawing of the final core-shell nanocolumnar thin film as well as cross-section scanning electron microscope (SEM) micrographs before and after ALD are depicted in Fig. 1. In order to determine the $\mathrm{Al}_{2} \mathrm{O}_{3}$ layer thickness, a $100 \mathrm{~nm}$ thick solid Co reference sample has been coated under identical ALD conditions. The resulting growth per cycle for the first 60 cycles determined with ellipsometry was $0.548 \AA$. Optical constants of transparent ALD grown $\mathrm{Al}_{2} \mathrm{O}_{3}$ have been determined prior to this investigation from an $18 \mathrm{~nm}$ thick layer with the Cauchy dispersion model. Angle-resolved spectroscopic Mueller matrix ellipsometry measurements were performed within a spectral range from 400 to $1000 \mathrm{~nm}$ (M2000VI, J. A. Woollam Co. Inc.). Spectra were taken at four different angles of incidence $\Phi_{a}=45^{\circ}, 55^{\circ}, 65^{\circ}$, and $75^{\circ}$, while performing a full sample rotation $0^{\circ} \leq \phi \leq 360^{\circ}$ in steps of $6^{\circ}$.

The experimentally acquired Mueller matrix spectra were analyzed with an augmented EMA approach based on the Bruggeman formalism. ${ }^{13}$ EMAs are physical models based on the properties and the relative fractions of its 
(a)

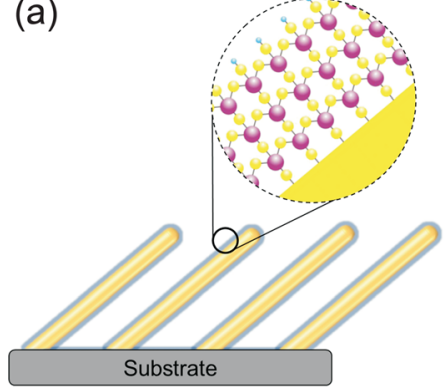

(b)

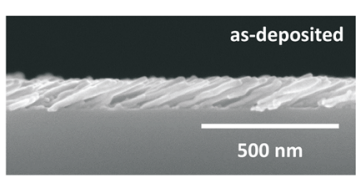

(c)

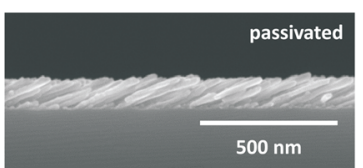

FIG. 1. (Color online) Schematic drawing of a core-shell Co SCTF coated with an $\mathrm{Al}_{2} \mathrm{O}_{3}$ passivation layer (3 ALD cycles depicted, (a)). Highresolution cross-section SEM micrographs reveal the structural equivalence before (b) and after (c) the ALD passivation process.

components and describe the macroscopic properties of a medium. The Bruggeman formalism, originally developed for spherical inclusions, is a homogenization process with absolute equality between the phases in mixture. An extension to randomly oriented anisotropic inclusions was obtained by introducing ellipsoidal depolarization factors $L_{a}^{\mathrm{D}}, L_{b}^{\mathrm{D}}, L_{c}^{\mathrm{D}}$, representative for the inclusions' polarization along major polarizability axes $\mathbf{a}, \mathbf{b}$, and $\mathbf{c}{ }^{14}$ The mixture macroscopically exhibits an isotropic effective polarizability $\left\langle\mathbf{P}_{\text {eff }}\right\rangle$ with a single (isotropic) effective dielectric function $\varepsilon_{\text {eff }}$ due to an averaging over all major polarizability axes (Fig. 2(a)). For spatially aligned inclusions of equal shape embedded in a host matrix (Fig. 2(b)), biaxial (orthorhombic) effective dielectric functions $\varepsilon_{\mathrm{eff}, a}, \varepsilon_{\mathrm{eff}, b}$, and $\varepsilon_{\mathrm{eff}, c}$ are obtained along $\mathbf{a}, \mathbf{b}$, and $\mathbf{c}$, respectively, instead of their average over all three semiaxes. ${ }^{6-8}$ The anisotropic Bruggeman EMA (AB-EMA) formulae for the three effective major dielectric functions $\varepsilon_{\mathrm{eff}, j}$ with $j=a, b, c$ for a mixture of $m$ constituents in implicit form are

$$
\sum_{n=1}^{m} f_{n} \frac{\varepsilon_{n}-\varepsilon_{\mathrm{eff}, j}}{\varepsilon_{\mathrm{eff}, j}+L_{j}^{\mathrm{D}}\left(\varepsilon_{n}-\varepsilon_{\mathrm{eff}, j}\right)}=0
$$

with the constraint that the sum of all material fractions $f_{n}$ equals unity. $\varepsilon_{n}$ is the dielectric function of the respective bulk material. $L_{j}^{\mathrm{D}}$ are the depolarization factors along the three orthogonal major polarizability axes $\mathbf{a}, \mathbf{b}$, $\mathbf{c}$, and the sum of all three depolarization factors must obey unity $\left(L_{a}^{\mathrm{D}}+L_{b}^{\mathrm{D}}+L_{c}^{\mathrm{D}}=1\right) \cdot{ }^{14,15}$ Note that in order to preserve the symmetry of the original Bruggeman theory, structural

(a)

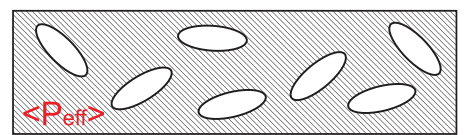

(b)

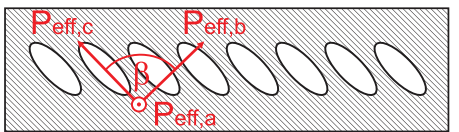

(c)

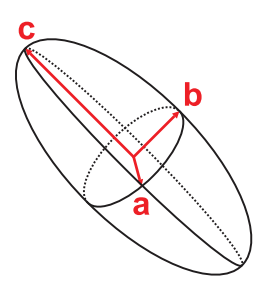

FIG. 2. (Color online) Effective medium scenarios with mixtures of ellipsoidal inclusions (general case) and a homogeneous host medium. The mixture with randomly oriented inclusions (a) exhibits an average effective polarizability $\left\langle\mathrm{P}_{\text {eff }}\right\rangle$ whereas the mixture with aligned inclusions (b) shows anisotropic properties with three effective polarizabilities $\mathrm{P}_{\mathrm{eff}, j}$. The major polarizability axes system rendering the biaxial nature of the film is depicted in (c).

equivalence between constituents is assumed (one set of $L_{j}^{\mathrm{D}}$ renders the effective medium geometry).

A real-valued rotation matrix $\mathbf{A}$ with wavelengthindependent Euler angles $\phi, \theta, \psi$ can then be found to transform the Cartesian laboratory coordinate frame into the material coordinate frame. ${ }^{16}$

As reported previously, considering the ensemble of slanted columns, the film exhibits monoclinic optical properties such that $\mathbf{P}_{\text {eff, } b}$ is not parallel to the semiaxis $b$ of the ellipsoid but rather tilted towards $c$ by a monoclinic angle $\beta .^{11,17,18}$ In order to describe biaxial materials with a monoclinic system, the obtained virtual orthogonal basis from Eq. (1) $\left(\varepsilon_{\text {eff }, j}=\varepsilon_{j}\right)$ has to be transformed by a projection matrix $\mathbf{U}:^{19}$

$$
\varepsilon_{\mathrm{m}}=\mathbf{U}\left(\begin{array}{ccc}
\varepsilon_{a} & 0 & 0 \\
0 & \varepsilon_{b} & 0 \\
0 & 0 & \varepsilon_{c}
\end{array}\right) \mathbf{U}^{\mathrm{T}} \quad \text { with } \mathbf{U}=\left(\begin{array}{ccc}
1 & 0 & 0 \\
0 & \sin \beta & 0 \\
0 & \cos \beta & 1
\end{array}\right),
$$

where $\beta$ is the monoclinic angle between axes $\mathbf{b}$ and $\mathbf{c}$.

Best-match model calculations are carried out by simultaneously varying relevant model parameters (see Table I) in order to match model calculated data as closely as possible to the experimental data sets.

Two measurements of the same sample (Co SCTF passivated with a thin $\mathrm{Al}_{2} \mathrm{O}_{3}$ coating) are compared, which have been conducted both immediately after deposition and again $60 \mathrm{~d}$ later. The stratified optical model is composed of three layers accounting for $\mathrm{Si}$ substrate, native $\mathrm{SiO}_{2}$, and an $\mathrm{AB}$ EMA layer for the SCTF. The best-match model parameters within the AB-EMA layer for both measurement scenarios are summarized in Table I (Ref. 20) and respective wavelength-by-wavelength determined optical constants depicted in Fig. 3.

The AB-EMA layer of the $\mathrm{Al}_{2} \mathrm{O}_{3}$ passivated Co SCTF comprises bulk-like optical constants of three constituents: $\mathrm{Co}$ (model parameters), $\mathrm{Al}_{2} \mathrm{O}_{3}$, and void $(n=1, k=0)$. Notably, except for material fractions, all best-match model parameters are virtually identical between measurements taken on the day of deposition and $60 \mathrm{~d}$ thereafter, respectively.

TABLE I. Overview of the best-match model parameters for both measurement scenarios, after deposition (0d) and 60 days later (60d), respectively, for the passivated Co SCTF. $t=$ thickness; $\theta=$ tilting angle with respect to the substrate normal; $\beta=$ monoclinic angle between axes $c$ and $a$; $f_{x}=$ volume fraction of $x ; L_{j}^{\mathrm{D}}=$ depolarization factor along $j$. The error limits given in parentheses denote the uncertainty of the last digit $(90 \%$ reliability).

\begin{tabular}{lcc}
\hline \hline Parameter & $\begin{array}{c}\mathrm{Co}+\mathrm{Al}_{2} \mathrm{O}_{3} \\
(\text { after deposition) }\end{array}$ & $\begin{array}{c}\mathrm{Co}+\mathrm{Al}_{2} \mathrm{O}_{3} \\
(60 \mathrm{~d} \text { later }\end{array}$ \\
\hline$t(\mathrm{~nm})$ & $89.46(3)$ & $89.86(3)$ \\
$\theta\left(^{\circ}\right)$ & $62.69(1)$ & $62.80(1)$ \\
$\beta\left(^{\circ}\right)$ & $82.96(2)$ & $83.39(2)$ \\
$f_{\mathrm{void}}(\%)$ & $62.03(7)$ & $59.33(6)$ \\
$f_{\mathrm{Al}_{2} \mathrm{O}_{3}}(\%)$ & $13.98(8)$ & $16.74(6)$ \\
$L_{a}^{\mathrm{D}}$ & $0.4035(2)$ & $0.4030(2)$ \\
$L_{b}^{\mathrm{D}}$ & $0.5267(2)$ & $0.5283(2)$ \\
$L_{c}^{\mathrm{D}}$ & $0.0698(7)$ & $0.0688(6)$ \\
$\mathrm{MSE}$ & 8.45 & 10.82 \\
\hline \hline
\end{tabular}



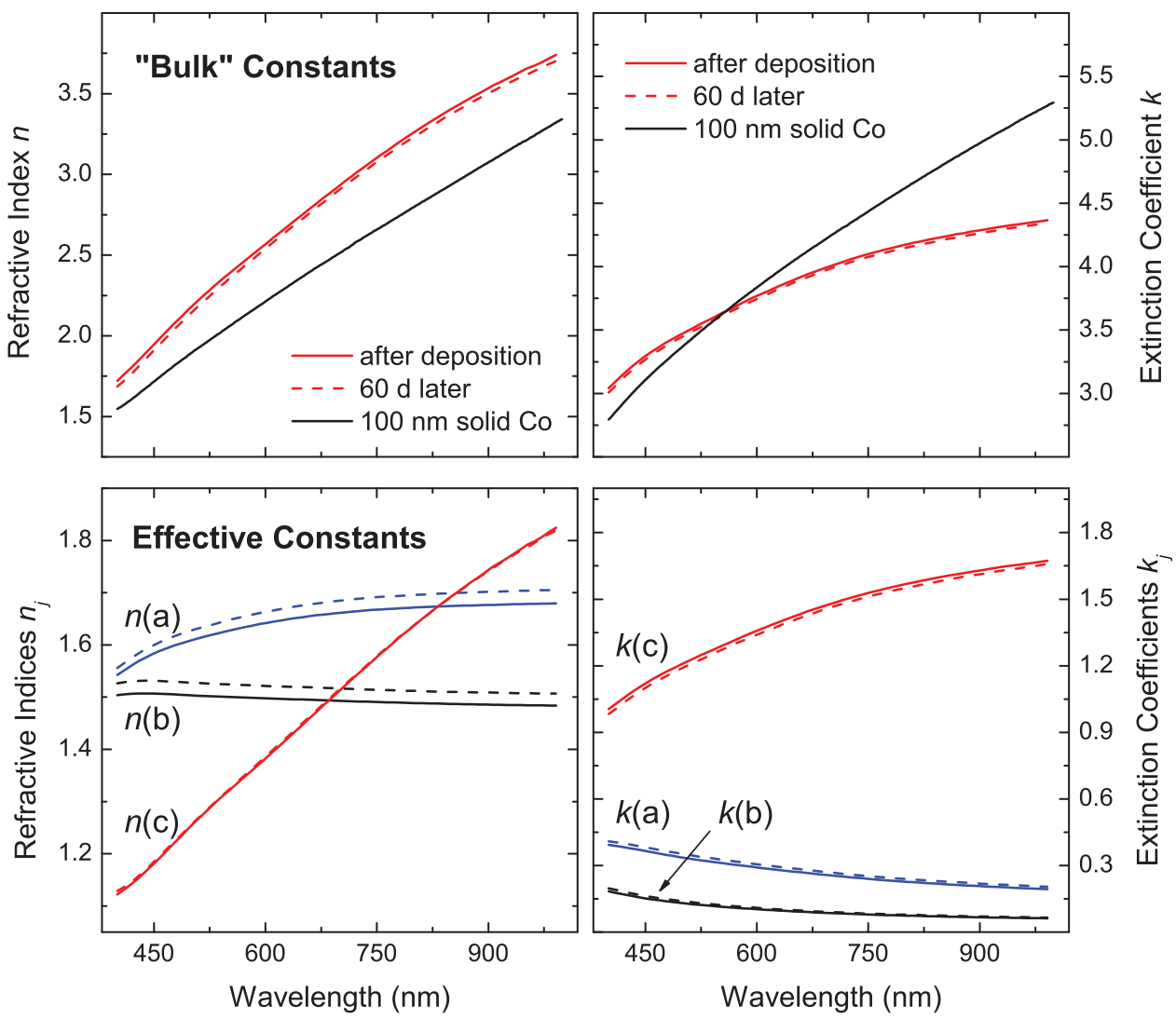

FIG. 3. (Color online) Wavelength-bywavelength determined optical constants for our $\mathrm{Al}_{2} \mathrm{O}_{3}$ passivated Co SCTF measured immediately after deposition (solid lines) and 60 days later (dashed lines), respectively. The first row shows the bulk-like optical constants $n$ and $k$, which are input parameters for the ABEMA approach and also compared to values obtained from a $100 \mathrm{~nm}$ solid Co thin film. The second row depicts the effective optical constants ( $n_{\text {eff }}$ and $\left.k_{\text {eff }}\right)$ along major axes of polarizability a, b, c, which are due to the different depolarization factors.
This shows that the film properties are not affected by storage in ambient air and expected aging effects due to material oxidation not present due to the $\mathrm{Al}_{2} \mathrm{O}_{3}$ passivation layer. The determined layer thickness $t$ and slanting angle $\theta$ are in very good agreement with cross-section SEM analysis (Fig. 1). The Co film fraction $f_{\text {Co }}$ is with $24 \%$ very low, which is characteristic for GLAD at very oblique angles. ${ }^{8}$ The observed imbalance between $f_{\text {void }}$ and $f_{\mathrm{Al}_{2} \mathrm{O}_{3}}$ as well as the slight changes in best-match Co bulk optical constants can be mainly attributed to differences in ambient humidity at the two measurement days. This is supported by the fact that there is virtually no effective optical constant variation along c. It is well-known that such nanostructured thin films are very sensitive high-speed capacitive humidity sensors. ${ }^{21}$ In general, the depolarization factors render a biaxial material with column-like geometry extended along the $c$-axis: $L_{c}^{\mathrm{D}} \ll L_{a}^{\mathrm{D}} \sim L_{b}^{\mathrm{D}}$. The wavelength-by-wavelength determined best-match Co bulk optical constants obtained from model analysis for the passivated Co SCTF after deposition and after $60 \mathrm{~d}$ are in excellent agreement with each other; however, differ from the ones determined for a $100 \mathrm{~nm}$ thin solid reference film grown in the same deposition chamber. This might be due to the idealized optical model as well as nanostructure confinement effects and the inherent large surface to volume ratio, which can affect the overall polarizability of the material. The effective optical properties only differ notably for $n_{\mathrm{eff}, a}$ and $n_{\mathrm{eff}, b}$, which indicates no material change due to oxidation ( $n_{\mathrm{eff}, c}$ unaffected), for example, but rather changes in the void regions caused by possible humidity changes. The investigation and quantification of water vapor adsorption in a controlled environment will be subject to a forthcoming publication.
The calculated $\mathrm{Al}_{2} \mathrm{O}_{3}$ shell layer thickness based on best-match AB-EMA constituent fraction parameters is with $2.4 \mathrm{~nm}$ in excellent agreement with the reference sample layer thickness of $3.29 \mathrm{~nm}$. Note that for this calculation, ideal and separated nanocolumns with an average diameter of $17.5 \mathrm{~nm}$ are assumed. High resolution SEM top-view and cross-section image analysis show that the diameter of individual columns is increased by approximately $4 \mathrm{~nm}$ after $\mathrm{ALD} \mathrm{Al}_{2} \mathrm{O}_{3}$ coating.

In summary, we have demonstrated that our generalized AB-EMA is an excellent approach to determine structural parameters as well as film fractions of multiple constituents of thin biaxial SCTFs with monoclinic optical properties. Furthermore, it was confirmed by spectroscopic Mueller matrix ellipsometry that a thin conformal $\mathrm{Al}_{2} \mathrm{O}_{3}$ ALD coating is suitable for passivation of metal SCTFs thereby preventing oxidation and aging effects. It is proposed that passivated three-dimensional sculptured thin films can be exploited as highly sensitive optical sensors, which may be analyzed by means of the presented AB-EMA approach to quantify sensed matter.

The authors acknowledge financial support from the National Science Foundation in RII (EPS-1004094) and CAREER (ECCS-0846329), the University of NebraskaLincoln, and the J. A. Woollam Foundation.

${ }^{1}$ O. Toader and S. John, Science 292, 1133 (2001).

${ }^{2}$ S. V. Kesapragada, P. Victor, O. Nalamasu, and D. Gall, Nano Lett. 6, 854 (2006).

${ }^{3}$ D. Schmidt, T. Hofmann, C. M. Herzinger, E. Schubert, and M. Schubert, Appl. Phys. Lett. 96, 091906 (2010).

${ }^{4}$ M. Knez, K. Nielsch, and L. Niinistö, Adv. Mater. 19, 3425 (2007). 
${ }^{5}$ O. Albrecht, R. Zierold, S. Allende, J. Escrig, C. Patzig, B. Rauschenbach, K. Nielsch, and D. Görlitz, J. Appl. Phys. 109, 093910 (2011).

${ }^{6}$ G. B. Smith, Opt. Commun. 71, 279 (1989).

${ }^{7}$ C. G. Granqvist, D. L. Bellac, and G. A. Niklasson, Renewable Energy 8, 530 (1996).

${ }^{8}$ G. Beydaghyan, C. Buzea, Y. Cui, C. Elliott, and K. Robbie, Appl. Phys. Lett. 87, 153103 (2005).

${ }^{9}$ R. A. May, D. W. Flaherty, C. B. Mullins, and K. J. Stevenson, J. Phys. Chem. Lett. 1, 1264 (2010).

${ }^{10}$ T. Hofmann, D. Schmidt, A. Boosalis, P. Kühne, R. Skomski, C. M. Herzinger, J. A. Woollam, M. Schubert, and E. Schubert, Appl. Phys. Lett. 99, 081903 (2011).

${ }^{11}$ D. Schmidt, A. C. Kjerstad, T. Hofmann, R. Skomski, E. Schubert, and M. Schubert, J. Appl. Phys. 105, 113508 (2009).

${ }^{12}$ C.-C. Wang, C.-C. Kei, Y.-W. Yu, and T.-P. Perng, Nano Lett. 7, 1566 (2007).

${ }^{13}$ D. A. G. Bruggeman, Ann. Phys. (Leipzig) 24, 636 (1935).
${ }^{14}$ D. Polder and J. H. van Santen, Physica 12, 257 (1946).

${ }^{15}$ A. Shivola, Electromagnetic Mixing Formulas and Applications, Electromagnetic Waves (The Institution of Electrical Engineers, London, 1999), Vol. 47.

${ }^{16} \mathrm{M}$. Schubert, Infrared Ellipsometry on Semiconductor Layer Structures: Phonons, Plasmons, and Polaritons, Springer Tracts in Modern Physics (Springer, Berlin, 2004), Vol. 209.

${ }^{17}$ D. Schmidt, B. Booso, T. Hofmann, E. Schubert, A. Sarangan, and M. Schubert, Appl. Phys. Lett. 94, 011914 (2009).

${ }^{18}$ D. Schmidt, B. Booso, T. Hofmann, E. Schubert, A. Sarangan, and M. Schubert, Opt. Lett. 34, 992 (2009).

${ }^{19}$ M. de Graef and M. E. McHenry, Structure of Materials: An Introduction to Crystallogrpahy, Diffraction, and Symmetry (Cambridge University Press, Cambridge, 2007).

${ }^{20}$ See supplementary material at http://dx.doi.org/10.1063/1.3675549 for further details on model parameter uniqueness.

${ }^{21}$ J. J. Steele, M. Taschuk, and M. J. Brett, IEEE Sens. J. 8, 1422 (2008). 